\title{
Branch graft patency after open repair of thoracoabdominal aortic aneurysms
}

\author{
Nicholas T. Kouchoukos, MD, ${ }^{\mathrm{a}}$ Alexander Kulik, MD, MPH, ${ }^{\mathrm{b}}$ and Catherine Castner, RN, BSN ${ }^{\mathrm{a}}$
}

\begin{abstract}
Objectives: The long-term function of branch grafts to the visceral and renal arteries during open thoracoabdominal aortic aneurysm repair is unknown. We assessed the patency of single and multiple branch grafts with postoperative imaging studies in patients followed for up to 13 years.

Methods: A total of 99 of 130 patients undergoing open thoracoabdominal aortic aneurysm repair who received a total of 298 branch grafts to the celiac, superior mesenteric, and renal arteries were evaluated with serial imaging studies at 6- to 12-month intervals. The mean duration of angiographic follow-up was 40.4 months and extended to 159 months. Thirty-three patients receiving 74 grafts were followed for more than 5 years, and 7 patents receiving 22 grafts were followed for more than 10 years. Eighty-four grafts were grafted to the celiac artery, 73 grafts were grafted to the superior mesenteric artery, 71 grafts were grafted to the left renal artery, and 70 grafts were grafted to the right renal artery.
\end{abstract}

Results: Nine graft occlusions occurred in 6 patients. One of these patients died of intestinal ischemia after occlusion of the celiac and superior mesenteric artery grafts, and 1 patient developed occlusion of both renal artery grafts and remains on dialysis. Five graft occlusions in the other 4 patients were asymptomatic, and no interventions were required. One additional patient developed significant stenosis of the celiac, superior mesenteric, and right renal arteries and underwent successful percutaneous angioplasty. No other patient required intervention. Freedom from occlusion of the 298 grafts at 1,5 , and 10 years is $98 \%, 97 \%$, and $93 \%$, respectively.

Conclusions: This represents the largest series of patients with branch grafts for open thoracoabdominal aortic aneurysm repair with extended angiographic follow-up. The favorable long-term graft patency rates represent a benchmark against which methods for establishing flow to the visceral and renal arteries using alternative techniques can be compared. (J Thorac Cardiovasc Surg 2017;153:S14-9)

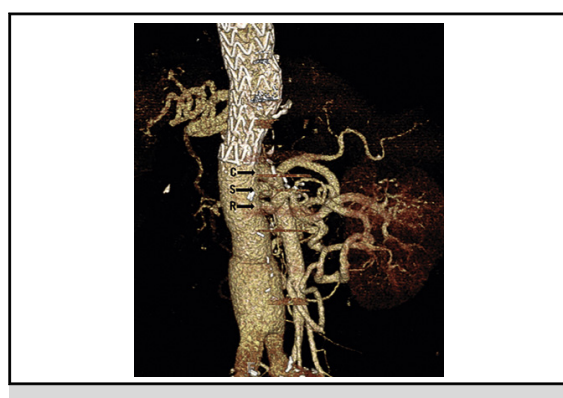

Patent branch grafts to the visceral and right renal arteries 129 months postoperatively.

\section{Central Message}

Long-term patency of branch grafts to the visceral and renal arteries after open TAAA repair exceeds $95 \%$ and is greater than patency after endovascular repair.

\section{Perspective}

Long-term angiographic follow-up of branch grafts to the visceral and renal arteries after open repair of TAAA demonstrates late patency rates that exceed $95 \%$. Intervention for branch graft stenosis was required in only 1 of 98 patients. These results represent a benchmark against which methods for establishing flow to the visceral and renal arteries using hybrid or endovascular techniques for TAAA repair can be compared.

See Editorial Commentary page S20.
During open repair of thoracoabdominal aortic aneurysms (TAAAs), several options are available for reattachment of the celiac artery, superior mesenteric artery (SMA),

From the ${ }^{\mathrm{a}}$ Division of Cardiovascular and Thoracic Surgery, Missouri Baptist Medical Center, BJC HealthCare, St Louis, Mo; and ${ }^{\mathrm{b}}$ Division of Cardiothoracic Surgery, Lynn Heart and Vascular Institute, Boca Raton Regional Hospital and Charles E. Schmidt College of Medicine, Florida Atlantic University, Boca Raton, Fla.

Read at the 2016 Annual Aortic Symposium of the American Association of Thoracic Surgeons, New York, New York, May 12-13, 2016.

Received for publication May 17, 2016; revisions received June 22, 2016; accepted for publication July 2, 2016; available ahead of print Aug 28, 2016.

Address for reprints: Nicholas T. Kouchoukos, MD, Cardiac, Thoracic, and Vascular Surgery Inc, 3009 N Ballas Rd, Suite 360C, St Louis, MO 63131 (E-mail: ntkouch@aol.com).

$0022-5223 / \$ 36.00$

Copyright (c) 2016 by The American Association for Thoracic Surgery

http://dx.doi.org/10.1016/j.jtcvs.2016.07.063 and renal artery. The inclusion technique, popularized by Crawford, ${ }^{1}$ involves the use of a side-to-side anastomosis between the prosthetic aortic graft and the aortic tissue surrounding the implanted vessel. The Carrel patch technique involves use of 1 or more full-thickness patches of aortic tissue containing the orifices of the aortic branches that are sutured to openings in the aortic graft in an end-to-side fashion. ${ }^{2}$ These techniques result in retention of aortic

Scanning this QR code will take you to the article title page. 


\section{Abbreviations and Acronyms \\ $\mathrm{CT}=$ computed tomography \\ SMA $=$ superior mesenteric artery \\ TAAA $=$ thoracoabdominal aortic aneurysm}

tissue that, over time, may be weakened from atherosclerotic degeneration or chronic aortic dissection. The potential exists for this tissue to become aneurysmal or to separate from the aortic graft, resulting in the development of false aneurysms or rupture. ${ }^{2-4}$ Previous studies indicate that the prevalence of visceral/renal artery patch aneurysms after TAAA repair using these techniques is between $4 \%$ and $8 \% .^{2,4-6}$

We previously used the Carrel patch technique during TAAA repair. ${ }^{7}$ However, because of the development of patch aneurysms and the subsequent availability of presewn single or multiple branch grafts, we modified our technique to attach the branches of these grafts directly to 1 or more of the visceral and renal arteries. ${ }^{8}$ Our early experience with 41 patients who received 141 branch grafts was favorable, demonstrating an actuarial patency rate of $98 \%$ at 5 years. ${ }^{9}$

With the advent and increasing use of endovascular techniques for repair of TAAA, information regarding the longterm function of grafts to the visceral and renal arteries with both open and endovascular methods has assumed increasing importance. In this context, we reviewed our entire experience with open TAAA repair using branch grafts to determine the durability and patency of the graft branches with follow-up that now extends to 13 years.

\section{MATERIALS AND METHODS \\ Patient Characteristics}

Between February 2001 and August 2015, 130 patients with Crawford extent I to IV TAAA underwent open surgical repair using cardiopulmonary bypass and hypothermic circulatory arrest. No other operative technique was used during this interval. Single or multiple collagenimpregnated branched grafts (Hemashield Platinum Branch Grafts: MAQUET Cardiovascular LLC, San Jose, Calif) were used to reimplant the visceral and renal arteries. ${ }^{7-10}$ The 30-day mortality was $6.9 \%$ (9 patients), and the hospital mortality was $9.2 \%$ (12 patients). Nonfatal postoperative complications included stroke $(5.4 \%, 7$ patients), paraparesis/ paraplegia $(10 \%, 13$ patients), and renal failure requiring dialysis $(10 \%$, 13 patients). Postoperative computed tomography (CT) scans or magnetic resonance angiogram studies with intravenous contrast were obtained before hospital discharge, when feasible, and at 6- to 12-month intervals during outpatient follow-up. Thirty-one patients did not undergo postoperative imaging studies with contrast because of hospital death (12 patients), early ( $<6$ months) out-of-hospital death ( 6 patients), and renal dysfunction that precluded imaging with intravenous contrast (5 patients). Of the remaining 107 patients, $99(92.5 \%)$ had imaging studies suitable for analysis and constitute the study group.

The clinical characteristics of the 99 patients are shown in Table 1. The mean age at operation was $63.5 \pm 14.5$ years (range, 15-87 years), and 54 patients were female $(54 \%)$. Sixteen patients $(16 \%)$ had clinical manifestations of Marfan, Loeys-Dietz, or Ehlers-Danlos syndrome. Thirty-six patients $(36 \%)$ had symptoms associated with their aortic disease. The
TABLE 1. Preoperative patient characteristics

\begin{tabular}{lc}
\hline \multicolumn{1}{c}{ Patient characteristic } & $\begin{array}{c}\text { No. of patients } \\
(\%)(\mathbf{n}=\mathbf{9 9})\end{array}$ \\
\hline Comorbidity & \\
Marfan syndrome/Ehlers-Danlos & $16(16)$ \\
Hypertension & $73(73)$ \\
History of smoking & $41(41)$ \\
Hyperlipidemia & $48(48)$ \\
Chronic obstructive pulmonary disease & $28(28)$ \\
Coronary artery disease & $30(30)$ \\
Peripheral vascular disease & $31(31)$ \\
Creatinine >1.5 mg/dL & $13(13)$ \\
Previous transient ischemic attack or stroke & $17(17)$ \\
Diabetes mellitus & $6(6)$ \\
Previous operations & \\
CABG or percutaneous coronary intervention & $25(25)$ \\
Aortic valve procedure & $25(25)$ \\
Ascending aortic repair & $36(36)$ \\
Aortic arch repair & $22(22)$ \\
Descending thoracic aortic repair & $10(10)$ \\
Thoracoabdominal aortic repair & $3(3)$ \\
Abdominal aortic aneurysm repair & $15(15)$ \\
Cause of aortic disease & \\
Degenerative aneurysm & $73(73)$ \\
Chronic dissection & $24(24)$ \\
Other & $2(2)$ \\
Aneurysm extent & \\
Crawford I & $7(7)$ \\
Crawford II & $45(45)$ \\
Crawford III & $45(45)$ \\
Crawford IV & $2(2)$ \\
\hline$C A B G$, Con
\end{tabular}

$C A B G$, Coronary artery bypass grafting.

remaining patients had aneurysms that were more than twice the size of adjacent normal aorta or had evidence of progressive aortic enlargement. Degenerative aneurysms were present in 73 patients, and 24 patients had chronic type A or type B dissections with aneurysms. Seven patients underwent a Crawford extent I TAAA repair, 45 patients underwent an extent II repair, 45 patients underwent an extent III repair, and 2 patients underwent an extent IV repair. The study was reviewed by the Institutional Review Board of the Missouri Baptist Medical Center and was exempt from board approval.

\section{Operative Technique}

Our general technique has been described. ${ }^{7-10}$ Cerebrospinal fluid drainage is used routinely, if technically feasible. A standard posterolateral thoracotomy incision is made and extended across the costal margin onto the left upper quadrant of the abdomen. The diaphragm is incised circumferentially. Cardiopulmonary bypass is established using the left femoral artery or a nondiseased segment of the descending thoracic aorta for arterial return. A long cannula is inserted into the left common femoral vein with the tip positioned in the right atrium for venous return. Cooling is initiated, and a catheter is placed into the left inferior pulmonary vein for venting of the left heart. When adequate cooling has been achieved (nasopharyngeal temperature $\leq 22^{\circ} \mathrm{C}$ ), circulatory arrest is established. A collagen-impregnated woven polyester graft of appropriate diameter containing a $10-\mathrm{mm}$ sidearm is sutured to the transected descending thoracic aorta or distal aortic arch. The side arm of the graft is then connected to a second arterial line from the pump-oxygenator. After evacuation of air, a clamp is placed on the graft just distal to the side arm and flow to the upper body is reestablished at a 
rate of 15 to $20 \mathrm{~mL} / \mathrm{kg}$ at a temperature of $20^{\circ} \mathrm{C}$ to $22^{\circ} \mathrm{C}$. Patent intercostal and lumbar arteries below the level of the sixth or seventh intercostal space are sutured to an opening in the graft when technically feasible, using a fullthickness cuff of aorta. The clamp on the graft is then repositioned below the intercostal pedicle to establish antegrade flow to the implanted arteries.

The distal thoracic and upper abdominal aortas are opened, and the orifices of the celiac artery, SMA, and renal artery are identified. If the aortic disease extends to the level of the iliac arteries, a knitted, collagen-impregnated bifurcation graft (Hemashield Platinum; MAQUET Cardiovascular LLC) is implanted. A clamp is placed on the body of the bifurcation graft or on the distal abdominal aorta to permit hypothermic perfusion of the lower body using the femoral artery cannula. The visceral and renal arteries are detached from the aorta with a small cuff of aortic tissue. If the origin of a vessel is severely stenotic or calcified, the vessel is transected beyond the aortic wall. The body of a 4-branched aortic graft is positioned so that the 3 adjacent vertically aligned branches lie opposite the origins of the celiac artery, SMA, and right renal artery. The distal end of the aortic branched graft is cut to the appropriate length and sutured to the infrarenal aorta or to a previously placed infrarenal graft. A clamp is placed on the graft below the branches. Direct perfusion of the visceral and renal arteries is not performed.

By maintaining hypothermic low flow, the anastomoses between the branches of the graft and the visceral and renal arteries are completed using 5-0 or 6-0 polypropylene sutures. ${ }^{8}$ Typically, the most distal $8-\mathrm{mm}$ branch is anastomosed to the right renal artery, followed by the middle $8-\mathrm{mm}$ branch to the SMA and the upper 10-mm branch to the celiac artery. The left renal artery is sewn to the fourth (perpendicular) $10-\mathrm{mm}$ branch or to a separate 6-mm or 8-mm graft that is then attached to the side of the aortic graft. After each anastomosis is completed, the distal clamp on the aortic graft is repositioned more proximally to enable perfusion of each implanted artery. In patients with Crawford extent I aneurysms, a single branch graft was used, attaching the branch to the celiac artery or SMA. Rewarming is initiated after establishing perfusion to all of the implanted arteries. The branched graft is then anastomosed to the descending thoracic aortic graft, and after evacuation of air from the grafts, full antegrade flow is established from the proximal arterial line.

A total of 298 grafts were used in the 99 patients. Fifty-six patients received 4 grafts, 12 patients received 3 grafts, 10 patients received 2 grafts, and 18 patients received 1 graft. Eighty-four grafts were placed to the celiac artery, 73 grafts were placed to the SMA, 71 grafts were placed to the left renal artery, and 70 grafts were placed to the right renal artery. The mean durations of cooling, circulatory arrest, low-flow hypothermic bypass, hypothermic ventricular fibrillation, rewarming, and cardiopulmonary bypass are shown in Table 2 . The mean duration of hypothermic circulatory arrest was $24.3 \pm 12.5$ minutes.

\section{Follow-up}

After hospital discharge, patients were evaluated clinically and with imaging studies 4 to 6 months postoperatively, and subsequently at 12-month intervals. The imaging studies were independently reviewed by the treating surgeon and a vascular radiologist. The date of last inquiry was February 2016 , and follow-up was $94 \%$ complete. The mean duration of angiographic follow-up was 40.4 months (range, 1-159 months), and the median duration was 28.1 months. Thirty-three patients who received 74 grafts were followed for more than 5 years, and 7 patients receiving 22 grafts were followed for more than 10 years. Patency of the visceral and renal branch grafts was defined as opacification of the branch lumen with contrast enhancement of the perfused organ. Stenosis was defined as more than $50 \%$ obstruction within the branch or the proximal native arterial lumen. Graft occlusion was defined as complete obstruction with no evidence of contrast within the branch lumen or enhancement of the perfused organ.

\section{Statistical Analyses}

Data were analyzed in Intercooled Strata 11.2 (StataCorp LP, College Station, Tex). Standard descriptive statistical analyses were used.
TABLE 2. Cardiopulmonary perfusion data

\begin{tabular}{lcl}
\hline & Mean \pm SD & Range \\
\hline Time (min) & & \\
Cardiopulmonary bypass & $173.5 \pm 36.6$ & $(105-307)$ \\
Cooling & $30.5 \pm 5.5$ & $(20-50)$ \\
Circulatory arrest & $24.3 \pm 12.5$ & $(0-76)$ \\
Low-flow hypothermic bypass & $65.8 \pm 30.0$ & $(14-160)$ \\
Hypothermic ventricular fibrillation & $112.2 \pm 35.5$ & $(45-230)$ \\
Spinal cord ischemia & $58.1 \pm 26.1$ & $(16-162)$ \\
Left renal ischemia & $98.4 \pm 35.7$ & $(19-179)$ \\
Right renal ischemia & $81.9 \pm 35.6$ & $(12-200)$ \\
Celiac ischemia & $106.7 \pm 36.0$ & $(40-215)$ \\
SMA ischemia & $91.4 \pm 36.3$ & $(30-230)$ \\
Rewarm & $71.9 \pm 15.5$ & $(35-135)$ \\
Temperature & & \\
Lowest nasopharyngeal & $15.4 \pm 2.0$ & $(10-22)$ \\
Lowest bladder/rectal & $21.2 \pm 2.8$ & $(14-28)$ \\
\hline
\end{tabular}

$S D$, Standard deviation; $S M A$, superior mesenteric artery.

Continuous data are presented as mean \pm standard deviation, and categoric data are presented as proportions. Nonparametric estimates of freedom from graft occlusion were determined using the Kaplan-Meier method and are reported as mean \pm standard error.

\section{RESULTS}

Nine graft occlusions occurred in 6 patients. One renal artery occluded early postoperatively, and 1 that was patent early postoperatively was occluded on the 6-month CT angiogram. The latter patient had severe stenosis of the renal artery preoperatively. Both patients had creatinine levels in the normal range at last follow-up. A 45-year-old woman with Marfan syndrome developed occlusion of the right renal artery 8 months after repair of a chronically dissected and aneurysmal thoracoabdominal aorta with a 4-branch graft. She underwent an iliac to renal artery bypass graft that occluded early postoperatively. The left renal artery occluded 2 months later, and repair was not attempted. She remains on permanent renal dialysis. A fourth patient

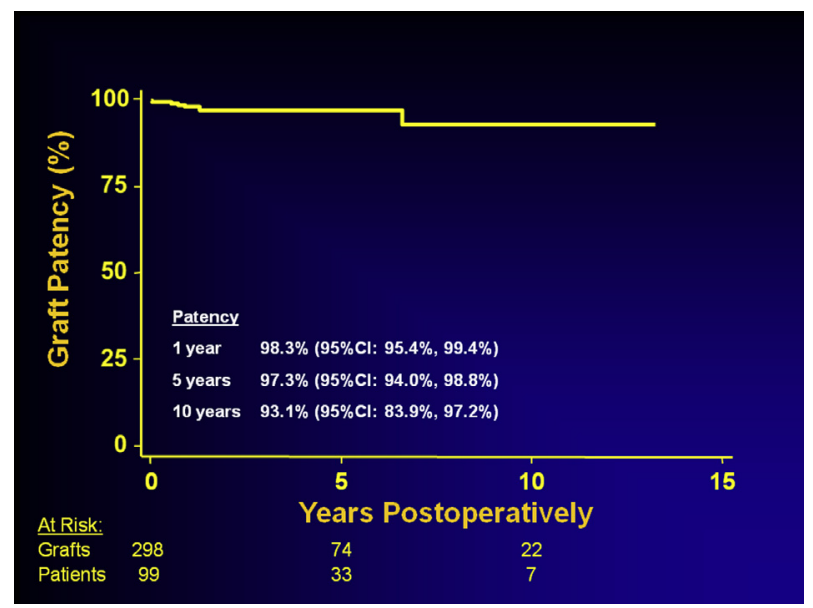

FIGURE 1. Patency of the visceral and renal artery branch grafts after TAAA repair. $C I$, Confidence interval. 
TABLE 3. Patency of the grafted visceral and renal arteries

\begin{tabular}{lrcc}
\hline Artery grafted & No. & No. occluded & Patency \\
\hline Celiac & 84 & 2 & $97.6 \%$ \\
SMA & 73 & 2 & $97.3 \%$ \\
Right renal & 70 & 2 & $97.1 \%$ \\
Left renal & 71 & 3 & $95.8 \%$ \\
Total & 298 & 9 & $97.0 \%$ \\
\hline
\end{tabular}

SMA, Superior mesenteric artery.

had occlusion of the left renal artery noted on a CT angiogram 18 months postoperatively. She has a normal creatinine level 2 years postoperatively. The fifth patient who received a 4-branch graft had a CT angiogram 13 months postoperatively that demonstrated patency of all 4 grafts. Two months later, she presented to the hospital with evidence of intestinal ischemia. A repeat CT angiogram demonstrated occlusion of the celiac and SMA branches and stenosis of the right renal artery branch. Her condition rapidly deteriorated, and she died shortly thereafter. The sixth patient was found by CT angiography to have occlusion of the SMA graft 74 months postoperatively and occlusion of the celiac artery 79 months postoperatively. These occlusions were not associated with symptoms, and the patient remains well 84 months postoperatively. Actuarial freedom from occlusion of the branch grafts to the renal and visceral arteries at 1 year, 5 years, and 10 years is $98.3 \% \pm 0.9 \%, 97.3 \% \pm 1.1 \%$, and $93.1 \% \pm 3 \%$, respectively (Figure 1). The absolute patency rates for the celiac artery, SMA, right renal artery, and left renal artery are
$97.6 \%, 97.3 \%, 97.1 \%$, and $95.8 \%$, respectively (Table 3, Figures 2 and 3).

An additional patient who received 4 grafts to the visceral and renal arteries developed $50 \%$ to $70 \%$ stenosis of the celiac artery, SMA, and right renal artery between the time of hospital discharge and the 6-month CT angiogram. Percutaneous angioplasty and stenting were successfully performed in all 3 branches. No other interventions have been performed to any of the branch grafts in the remaining patients. One patient has mild narrowing of the left renal artery graft but has normal renal function. One patient has mild stenosis of a celiac artery branch but no symptoms of intestinal ischemia. A third patient has mild narrowing of a renal artery graft and the adjacent renal artery, and has developed progressive atrophy of the kidney that was noted 8 months postoperatively (last creatinine $=2.2 \mathrm{mg} / \mathrm{dL}$ ). An additional patient with a patent renal artery graft required a nephrectomy for a renal tumor.

\section{DISCUSSION}

To the best of our knowledge, this study represents the largest series of patients with aortic branch grafts undergoing open repair of TAAA with extended angiographic follow-up to assess patency. The absolute patency rates of separate branch grafts to the visceral and renal arteries are excellent, ranging from $97.6 \%$ to $95.7 \%$, with an actuarial patency rate for all grafts of $93 \%$ at 10 years. Only 2 of 141 grafts followed for more than 2 years have occluded, and only 3 patients have mild narrowing of branch grafts
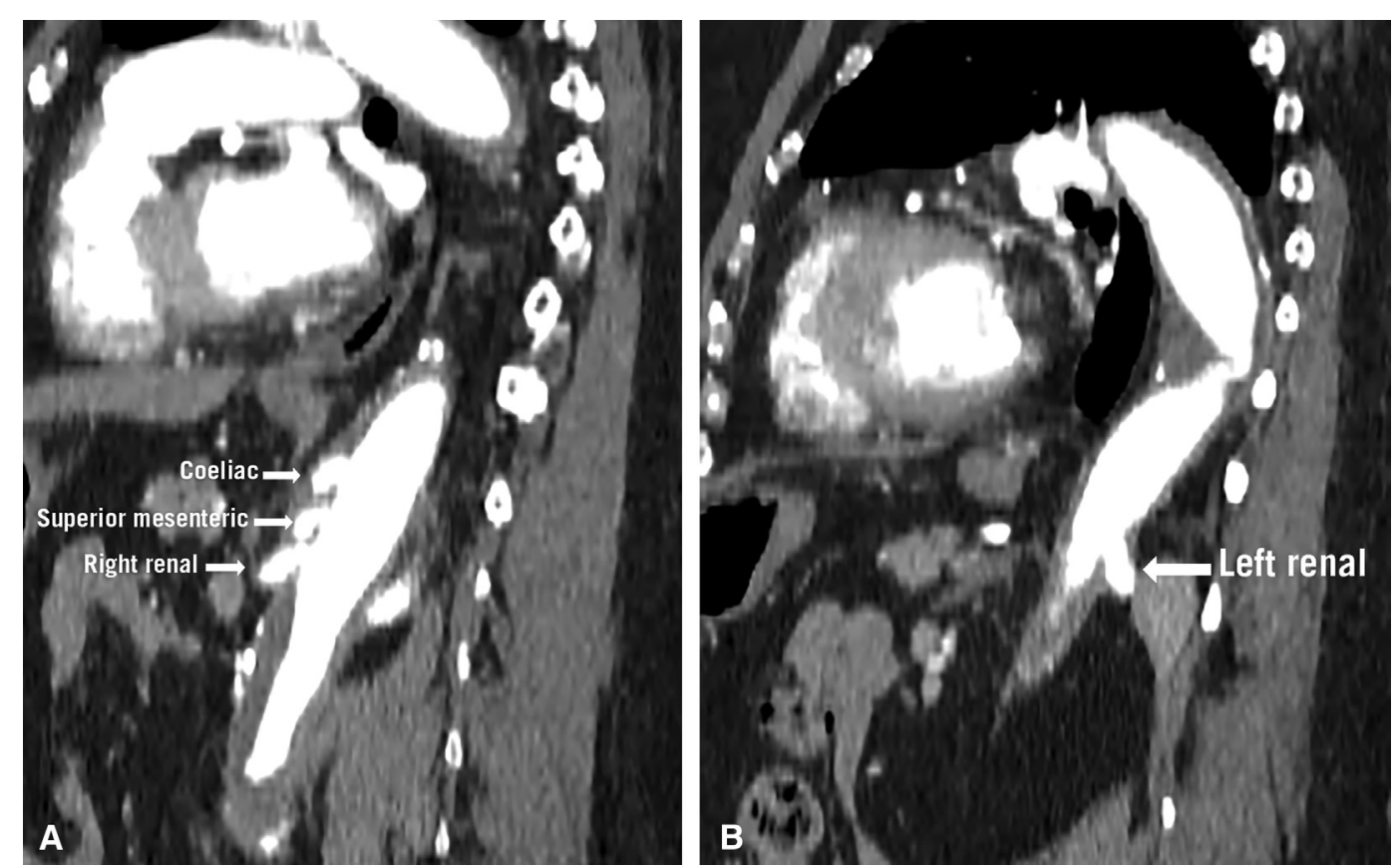

FIGURE 2. A, CT angiogram demonstrating patency of branches to the celiac artery, SMA, and right renal artery (arrows) 139 months after repair of a Crawford extent II degenerative TAAA. B, Patency of branch graft to the left renal artery (arrow). 


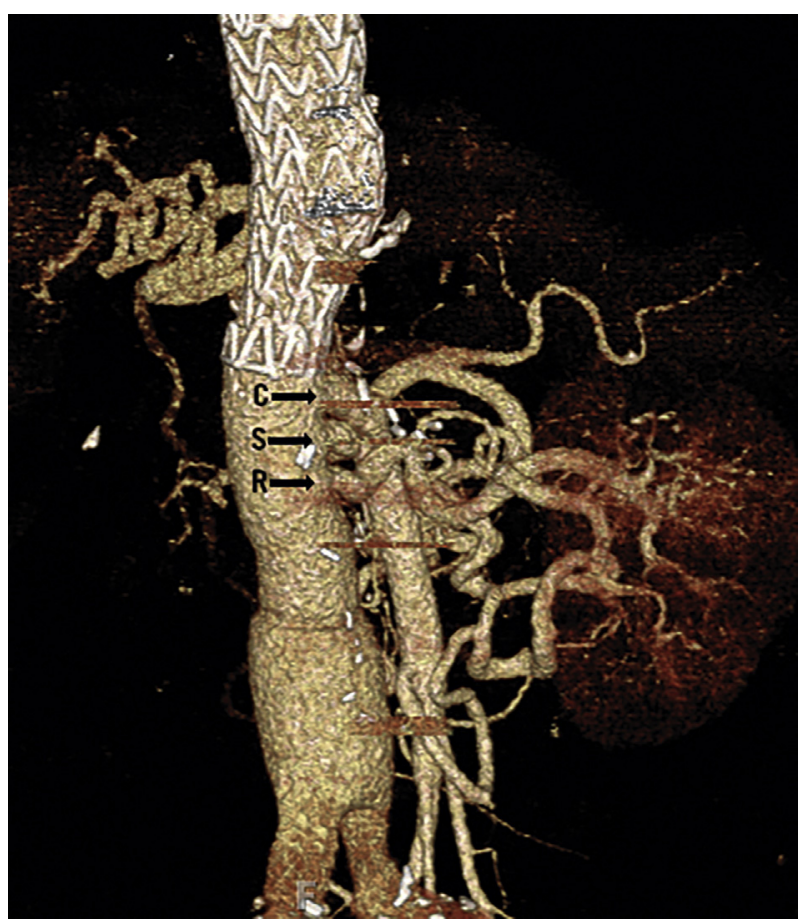

FIGURE 3. CT angiogram demonstrating patency of branch grafts to the celiac artery $(C)$, SMA $(S)$, and right renal artery $(R) 129$ months after repair of a Crawford extent II chronic dissecting TAAA. The left renal artery occluded at the time of the initial acute dissection. The stent graft in the descending thoracic aorta was placed to treat an intercostal artery patch aneurysm.

( 2 renal, 1 celiac) that have not required intervention. We are aware of only 1 other study that has evaluated late patency of branch grafts after TAAA repair. Youssef and colleagues ${ }^{6}$ assessed the patency of 12 branch grafts ( 3 celiac, 2 right renal, 7 left renal). All grafts remained patent at a median follow-up interval of 66 months. ${ }^{6}$ These arteries were selectively grafted when the island patch technique could not be used.

Developments in endovascular stent-graft technology have led to increasing application of hybrid debranching strategies and use of branched and fenestrated stent grafts for TAAA repair. Among recently reported series that used hybrid procedures, primary patency rates of the bypassed visceral and renal branches within the first several years of follow-up ranged between $90 \%$ and $95 \% .{ }^{11-14} \mathrm{In}$ a series of 45 patients with longer follow-up, Massoni and colleagues ${ }^{14}$ reported primary graft patency rates at 1,2 , and 4 years of $89.7 \%, 85.3 \%$, and $79 \%$, respectively. With long-term patency rates that are far from ideal, the need for early and late reintervention with these techniques continues to be an important issue. The rates of early reintervention in 3 of the series ranged from $8 \%$ to $54 \%$. ${ }^{11,13,14}$ In the series of Massoni and colleagues, ${ }^{14}$ freedom from reintervention at 1,2 , and 4 years was $45.6 \%, 45.6 \%$, and $34.2 \%$, respectively.
The largest study assessing the durability of branched and fenestrated endografts for TAAA repair has been reported from the Cleveland Clinic. ${ }^{15} \mathrm{~A}$ total of 650 patients received 1679 branch grafts to the visceral and renal arteries. During the follow-up interval that averaged 3 years and extended to 9 years, secondary procedures were performed for $0.6 \%$ of celiac artery, $4 \%$ of SMA, $6 \%$ of right renal artery, and 5\% of left renal artery branches. The mean time to intervention was 237 days. The 30-day, 1-year, and 5-year rates of freedom from branch intervention were $98 \%, 94 \%$, and $84 \%$, respectively. The interventions were performed for endoleak or development of occlusion or stenosis of the branch grafts.

Troisi and colleagues ${ }^{16}$ evaluated 107 patients who received fenestrated or branch grafts for repair of TAAA and suprarenal and pararenal aneurysms. Thirty-four secondary procedures were performed in 28 patients $(26.2 \%)$. The majority of the interventions were for stenosis or occlusion of the branch grafts and for type III endoleaks. The 3-year freedom from any device-related secondary procedure was $75.5 \%$. Premprabha and colleagues ${ }^{17}$ evaluated 100 patients who received multibranched aortic stent-grafts. During a follow-up interval that averaged 25.6 months, they observed no SMA branch occlusions (0/100), 2 of 95 celiac artery occlusions $(2.1 \%)$, and 18 of 187 renal branch occlusions $(9.6 \%)$. In all of these series, and in the series of Fernandez and colleagues, ${ }^{18}$ the prevalence of occlusion of renal artery grafts was substantially higher than for grafts to the celiac artery and SMA. ${ }^{15-17}$ Troisi and colleagues ${ }^{16}$ and O'Callaghan and colleagues ${ }^{19}$ have drawn attention to stent migration and development of type I endoleaks, which have resulted in destabilization of the stent-graft repairs. In the report by O'Callaghan and colleagues, ${ }^{19}$ development of type I endoleaks was associated with increased component instability and aortic related death. The time to endoleak occurrence ranged from the early postoperative period up to 4 years after the procedure.

Overall, the data from our study suggest favorable longterm durability of branch grafts to the visceral and renal arteries after open repair of TAAA. In the absence of large randomized trials, it is obviously difficult to fairly compare the perioperative outcomes of open versus stent-grafting procedures for TAAA repair, given the differing baseline characteristics of the patients and the varying techniques used from center to center. However, in terms of late outcomes, we believe that a fair comparison can be made between open and endovascular approaches, because patient characteristics are unlikely to substantially influence late postoperative graft patency. Our study indicates that after open operation, the long-term patency of branch grafts is at least equivalent and likely superior to that for the currently used endovascular procedures. This seems particularly true for grafts placed to the renal arteries. Late graft occlusion or stenosis remains a concern with stent-grafting 
techniques, and the frequency of subsequent interventions to correct these problems is not insignificant. The frequency of intervention after open repair is substantially lower, and late occlusion or development of important stenosis is exceedingly rare. We believe our data can serve as a benchmark against which the long-term function of methods for establishing flow to the visceral and renal arteries using endovascular or hybrid techniques can be compared. Because complications can develop late after TAAA repair, we continue to advocate for annual surveillance with imaging even among patients who are asymptomatic.

\section{Study Limitations}

This study is the largest series to date to report long-term durability data after open TAAA repair using branch grafts. However, the results must be interpreted within the context of the study design. It involved retrospective review of prospectively collected data in a referral-based tertiary care center. Many patients were referred to our center from afar. However, every effort was made to obtain imaging surveillance at regular intervals, even from a distance. Because of early death, postoperative renal dysfunction, or inability to obtain postoperative imaging studies, patency data could not be obtained for some patients who were excluded from the study.

\section{CONCLUSIONS}

Our study represents the largest series to date of patients with branch grafts for open TAAA repair who have undergone extended imaging follow-up to assess long-term graft durability. The favorable patency and low rate of reintervention for graft failure represent a benchmark against which endovascular and hybrid techniques for TAAA repair can be compared.

\section{Conflict of Interest Statement}

Authors have nothing to disclose with regard to commercial support.

\section{References}

1. Crawford ES. Thoraco-abdominal and abdominal aortic aneurysms involving renal, superior mesenteric, celiac arteries. Ann Surg. 1974;179:763-72.
2. Dardik A, Perler BA, Roseborough GS, Williams GM. Aneurysmal expansion of the visceral patch after thoracoabdominal aortic replacement: an argument for limiting patch size? J Vasc Surg. 2001;34:405-10.

3. Dias RR, Coselli JS, Stolf NA, Dias AR, Mady C, Oliveira SA. Aneurysmal dilation of the reimplant segment of the visceral vessels after thoracoabdominal aneurysm correction [Article in English, Portugues]. Arq Bras Cardiol. 2003; 81:273-8

4. Tshomba Y, Melissano G, Civilini E, Setacci F, Chiesa R. Fate of the visceral aortic patch after thoracoabdominal aortic repair. Eur J Vasc Endovasc Surg. 2005;29:383-9.

5. LeMaire SA, Carter SA, Volguina IV, Laux AT, Milewicz DM, Borsato GW, et al Spectrum of aortic operations in 300 patients with confirmed or suspected Marfan syndrome. Ann Thorac Surg. 2006;81:2063-78.

6. Youssef M, Neufang A, Jungmann F, Vahl C-F, Dorweiler B. Patency of renal and visceral vessels after open thoracoabdominal aortic replacement. J Vasc Surg. 2015;62:594-9.

7. Kouchoukos NT, Masetti P, Rokkas CK, Murphy SF, Blackstone EH. Safety and efficacy of hypothermic cardiopulmonary bypass and circulatory arrest for operations on the descending thoracic and thoracoabdominal aorta. Ann Thorac Surg. 2001;72:699-708.

8. Kouchoukos NT, Masetti P, Castner CF. Use of presewn multiple branched graft in thoracoabdominal aortic aneurysm repair. J Am Coll Surg. 2005;201:646-9.

9. Kulik A, Castner CF, Kouchoukos NT. Patency and durability of presewn multiple branched graft for thoracoabdominal aortic aneurysm repair. J Vasc Surg. 2010;51:1367-72.

10. Kulik A, Castner CF, Kouchoukos NT. Outcomes after thoracoabdominal aortic aneurysm repair with hypothermic circulatory arrest. J Thorac Cardiovasc Surg. 2011;141:953-60.

11. Patel R, Conrad MF, Paruchuri V, Kwolek CJ, Chung TK, Cambria RP. Thoracoabdominal aneurysm repair: hybrid versus open repair. J Vasc Surg. 2009; $50: 15-22$.

12. Kabbani LS, Criado E, Upchurch GR Jr, Patel HJ, Eliason JL, Rectenwald J, et al Hybrid repair of aortic aneurysms involving the visceral and renal vessels. Ann Vasc Surg. 2010;24:219-24.

13. Patel HJ, Upchurch GR Jr, Eliason JL, Criado E, Rectenwald J, Williams DM et al. Hybrid debranching with endovascular repair for thoracoabdominal aneurysms: a comparison with open repair. Ann Thorac Surg. 2010;89:1475-81.

14. Massoni CB, Geisbüsch P, Gallitto E, Hakimi M, Gargiulo M, Böckler D. Followup outcomes of hybrid procedures for thoracoabdominal aortic pathologies with special focus on graft patency and late mortality. J Vasc Surg. 2014;59:1265-73.

15. Mastracci TM, Greenberg RK, Eagleton MJ, Hernandez AV. Durability of branches in branched and fenestrated endografts. J Vasc Surg. 2013;57:926-33.

16. Troisi N, Donas KP, Austermann M, Tessarek J, Umscheid T, Torsello G. Secondary procedures after aortic aneurysm repair with fenestrated and branched endografts. J Endovasc Ther. 2011;18:146-53.

17. Premprabha D, Sobel J, Pua C, Chong K, Reilly LM, Chuter TA, et al. Viscera branch occlusion following aneurysm repair using multibranched thoracoabdominal stent-grafts. J Endovasc Ther. 2014;21:783-90.

18. Fernandez CC, Sobel JD, Gasper WJ, Vartanian SM, Reilly LM, Chuter TAM, et al. Standard off-the-shelf versus custom-made multibranched thoracoabdominal aortic stent grafts. J Vasc Surg. 2016;63:1208-15.

19. O'Callaghan A, Greenberg RK, Eagleton MJ, Bena J, Mastracci TM. Type Ia endoleaks after fenestrated and branched endografts may lead to component instability and increased aortic mortality. J Vasc Surg. 2015;61:908-14.

Key Words: thoracoabdominal aorta, open repair, branch grafts 